\title{
Influenza: Preparedness for an Inevitable "Emergency" for Oncology and BMT Units
}

John R. Wingard, MD, Gainesville, Florida

Key Words

Influenza, pandemic, emergency preparedness

\begin{abstract}
Influenza is a seasonal and pandemic threat to the general population. The effects can be devastating for cancer patients and those who have undergone blood and marrow transplantation (BMT). However, this impact can be minimized. Emergency preparedness is the key to mitigating the impact on the oncology and BMT services. Having a plan that provides a framework of preparedness and outlines steps to take in the event of a community outbreak is crucial. A vigorous immunization campaign for patients, household contacts, and health care staff early in the season is the single most important action. In the midst of an outbreak, the oncology and BMT teams should act to quickly identify patients with suspected infections, move infected patients and staff away from noninfected patients to prevent contact, and decide which patients require prevention or treatment with antiviral agents. Ongoing engagement by the entire team to evaluate the effectiveness of its actions and modify its plan as necessary will ensure success. (JNCCN 2008;6:215-222)
\end{abstract}

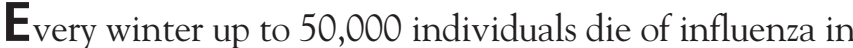
the United States. ${ }^{1-3}$ Many of these deaths are avoidable. In addition, tens of millions of work and school days are lost and hundreds of thousands of excess hospitalizations occur annually because of influenza illnesses. However, despite public health campaigns that recommend vaccination and other measures to protect the public, the United States repeatedly fails to do all that can be done.

From Division of Hematology/Oncology, University of Florida College of Medicine, Gainesville, Florida.

Submitted August 26, 2007; accepted for publication December 11, 2007.

The author is a consultant for Pfizer and Merck, and is on the speakers' bureau for Pfizer, Merck, Schering-Plough, and MGI Pharma.

Correspondence: John R. Wingard, MD, Division of Hematology/ Oncology, University of Florida College of Medicine,

P.O. Box 103633, 1376 Mowry Road, Gainesville, FL 32610-3633.

E-mail: wingaj@@medicine.ufl.edu
Moreover, every 3 to 4 decades, influenza seems to cause pandemics that can result in up to tens of millions of deaths worldwide. ${ }^{4,5}$ If this trend continues, the next pandemic is overdue. Avian influenza outbreaks have occurred in several countries, with a case fatality rate exceeding 50\%. The concept of a potential influenza pandemic is real, and the World Health Organization (WHO) warns of the possibly devastating consequences. ${ }^{6}$ This article discusses the specific issues practitioners encounter for cancer patients and those who have undergone bone marrow transplantation (BMT) and how health care teams can prepare to minimize the potential harm from influenza.

\section{Threat of Seasonal and Pandemic Influenza}

Influenza viruses are enveloped single-stranded RNA viruses that are members of the Orthomyxoviridae family. Three types exist: influenza A, B, and C, with most human disease caused by types $\mathrm{A}$ or B. Type A viruses account for most annual seasonal outbreaks, whereas type $B$ viruses cause seasonal outbreaks at 2-to 4-year intervals. Sometimes, more than 1 type or strain may circulate in the population at the same time. Pandemics are caused by type A strains. Subtypes are categorized on the basis of 2 types of surface glycoproteins that have hemagglutinin and neuraminidase activity. Various hemagglutinin and neuraminidase glycoproteins have been identified that are antigenically distinct. The surface hemagglutinin and neuraminidase glycoproteins induce antibody responses in humans, whereas internal viral proteins may induce cytotoxic T-cell responses. Immunity provides protection from severe illness.

Despite immunity gained from either exposure to influenza or immunization, new infections and illness from influenza viruses continue to occur because the viruses change continually. Influenza viruses cause outbreaks in 
2 general ways: 1) proofreading errors by the viral RNA polymerase lead to new variants of hemagglutinin and neuraminidase glycoproteins that are slightly different, and these new antigens offer advantages to new strains over older influenza strains because of less resistance in the population; and 2) coinfection of a host cell by 2 different influenza viruses (e.g., an animal strain and a human strain), which allows recombination of individual segments of the viral RNA, and the "reassorted" virus can express novel proteins different from those expressed by previous strains and against which the population may have little or no resistance. In both situations, the variations allow evasion of whatever preexisting humoral immunity may exist and facilitate an outbreak in a community. In general, the frequent, minor antigenic changes (antigenic shifts) that occur from year to year cause most of the annual winter outbreaks, whereas the much less frequent major antigenic changes (antigenic drifts), to which the general population has virtually no immunity, give rise to more deadly pandemics. As new variants occur through antigenic drift, establish niches in the population, and circulate more widely, they gradually replace older strains. Immunity to newer strains in the population builds with successive seasons. However, this creates a favorable scenario for a new, highly virulent virus that may arise through antigenic shift, to which the population has no preexisting immunity, thereby allowing it to circulate easily. These ebbs and flows occur cyclically, and influenza pandemics occur at roughly 10 - to 40-year intervals. Past death tolls from influenza pandemics have been measured in the millions worldwide. With global travel, international trade patterns, and continuously increasing populations in densely populated urban centers, the risk for a worldwide disaster is real.

Transmission among humans occurs primarily through respiratory droplets containing infectious virions. After a susceptible person inhales infectious virus, an incubation period of 1 to 2 days occurs before onset of symptoms. Shedding of virus from infected patients begins approximately 24 hours before symptoms develop, is heaviest during the first 2 days of acute symptomatic illness, then falls dramatically over 5 to 10 days. More prolonged shedding may occur in healthy children and immunocompromised patients. In patients who have undergone $\mathrm{BMT}^{8}{ }^{8}$ a correlation may exist between the number of infectious virions shed and severity of illness from various respiratory viruses.
Influenza manifests clinically primarily as an upper respiratory infection. Initially, systemic symptoms such as high fever, myalgias, headache, and lassitude predominate, followed 2 to 3 days later by dry cough, oropharyngeal pain, and nasal discharge in individuals who are not immunocompromised. Two major complications that can occur are viral pneumonia and secondary bacterial pneumonia.

Oncology and BMT services have unique vulnerabilities to influenza. The influenza syndrome is similar in patients with cancer and those who have undergone BMT, but high fever and myalgias may be much less prominent compared with healthy individuals. Because of their impaired host defenses, oncology patients are more likely to have a mild illness progress to pneumonia and have a greater risk for death. ${ }^{9-13}$ Various case series involving patients with leukemia and those who underwent BMT have shown that lower respiratory tract involvement can occur in $15 \%$ to $30 \%$ of infected patients. ${ }^{14}$ The degree of risk for influenza complications is influenced by the depth of immunosuppression. Children with cancer (especially hematologic malignancies), adults with acute leukemia, and BMT recipients are most susceptible to influenza complications. ${ }^{15-18}$ Factors associated with a greater risk for progression to pneumonia include older age ( $>65$ years) and the presence of severe neutropenia or lymphopenia. Among patients who develop pneumonia, severe lymphopenia (absolute lymphocyte count $<200$ cells $/ \mathrm{mL}$ ) is associated with a higher risk for death. ${ }^{14}$ Death has been noted in 15\% to 30\% of patients with pneumonia. Even in survivors of influenza, residual sequelae may include long-lasting pulmonary impairment. ${ }^{19,20}$ Nosocomial transmission from infected health care workers and visitors to patients can be a major problem in cancer units. ${ }^{21}$ Viral shedding may last longer and higher titer virus particles may be shed in patients with cancer and those who have undergone BMT. ${ }^{22,23}$

Diagnosis can be achieved through culture or detection of viral antigens in respiratory secretions. Recovery of virus is less likely from throat swabs than from other respiratory samples, such as nasopharyngeal swabs, which provide a higher quantity of virus. Appropriate collection, prompt transport, and laboratory processing are important requirements of cultures. Cultures usually become positive within 3 days. Tests detecting viral antigens are more rapid, with results available in less than 1 hour, but sensitivities 
have varied and depend on the concentration of virus in the sample. Polymerase chain reaction assays also provide rapid diagnosis. The sensitivities of the various rapid diagnostics have been $70 \%$ to $75 \%$, with specificities of $90 \%$ to $95 \%$. Some experts advocate an epidemiologic diagnosis. When influenza is circulating in the community, acute influenza-like symptomatology (especially cough and fever) in individuals without underlying respiratory disease likely indicates influenza infection. ${ }^{24}$ Some experts suggest initiating anti-influenza therapy based on this presumption, without laboratory confirmation, to optimize early initiation of therapy. This may be particularly useful in practice settings that do not have easy access to rapid diagnostic assays.

Over the past 300 years, 10 influenza A pandemics have occurred. Pandemics in the twentieth century occurred in 1918, 1957, and 1968. Thirty-nine years have elapsed since the last pandemic, and the end of an interpandemic period is approaching, according to many experts. Herald events occurred before the previous pandemics, and the recent skirmishes with a new variant of an avian influenza strain show many hallmarks of an imminent influenza pandemic. ${ }^{25}$ The WHO has issued an alert indicating that the United States is currently in phase 3 of a pandemic (i.e., a threat is looming but extensive human-to-human spread has not yet occurred).

Animal reservoirs are important sources of new variants of influenza, especially fowl and swine. Close contact between animals and humans allows opportunities for animal strains to infect humans. Wet markets in Southeast Asia, in which both fowl and swine are placed in the midst of large urban populations, provide ideal conditions for spread to humans. Through reassortment of viral RNA, a new strain may be produced that can cross species, such as fowl to humans, and transmit efficiently to other humans.

Current concern exists about the avian influenza A strain (H5N1), which many experts believe has the capacity to cause a pandemic. H5N1 influenza was first recognized in 1997 when human cases were noted in association with a poultry outbreak in Hong Kong, where 1 in 3 infected humans died. ${ }^{26}$ Other poultry outbreaks have been noted in recent years. Cumulatively, more than 300 humans have been infected as of July 2007 , with a staggering case fatality rate of $60 \%$ and most deaths occurring within 2 weeks from progressive respiratory failure. Updates of the current situation are available on the WHO Web site (www.who.int/csr) disease/avian_influenza/country/en/). matory cytokines and chemokines seems to contribute to the high degrees of morbidity and mortality. ${ }^{27}$ Fortunately, human-to-human transmission of current $\mathrm{H} 5 \mathrm{~N} 1$ strains remains poor, although this all can abruptly change through viral RNA reassortment events typical of historical pandemics. ${ }^{28}$ The likelihood that a localized outbreak will spread is substantial because novel strains are easily spread through migratory birds and air travel by humans.

\section{Elements of Emergency Planning}

Preparedness for seasonal and pandemic influenza must begin long before an outbreak. To be effective, international, national, and local community efforts must be established and their execution coordinated.

Special considerations for patients with cancer and those who have undergone BMT necessitate an emergency preparedness program for the services that address the concerns of these patients. Cancer treatment delays may be necessary to avoid initiating antineoplastic therapy or starting BMT conditioning regimens in patients with active illness. Infected family members or caregivers may not be able to assist these patients. Blood supplies may be interrupted when substantial numbers of donors become infected. Absenteeism among health care providers may reduce the staff needed to care for patients. Hospital beds may not be available for cancer care, neutropenia from chemotherapy, or other neoplastic complications requiring hospitalization, because they are occupied by patients infected with influenza.

An emergency preparedness framework for the BMT service has been suggested ${ }^{29}$ that is applicable for both oncology and BMT services. This is applicable for not only seasonal and pandemic influenza but also for other disasters (e.g., earthquake, weather event, blackout) oncology teams may encounter.

At the center of emergency preparedness is a plan that addresses steps of mitigation, preparedness, response, and recovery. Mitigation refers to efforts to determine how a service would be impacted and taking steps to address that impact. Identifying key individuals, establishing an emergency preparedness team, creating a command structure, and preparing communications links are the first tasks. Assessing any weaknesses in the inpatient and outpatient capacity is 
important. Identifying unique oncology needs (and those that will not be addressed in the larger institutional or community plans) and specific local concerns is also important.

Preparedness refers to staff training for an emergency, including practice drills, inspection of supplies, evaluation of surge capacity, and ordering of extra supplies (e.g., antivirals, antibiotics, masks, vaccines).

Response refers to the team's actions once an outbreak has started. The team's actions must be flexible to events as they unfold and be coordinated with institutional and community actions. Particularly important are actions necessary to keep infected patients (and staff) away from noninfected individuals.

Recovery refers to measures taken to restore the service to normal after the emergency has subsided. Cancer treatment plans for individual patients must be resumed. The staff may have psychological issues to address, and some members may need respite. The team should assess what steps worked and which did not and modify the plan as needed for future influenza outbreaks.

\section{Planning for Influenza}

\section{Vaccination}

The major strategy for influenza prevention is vaccination. ${ }^{30}$ Two types of vaccines are highly effective. A live attenuated vaccine is indicated for healthy individuals aged 5 to 49 years, but should be avoided by patients with impaired immunity and health care workers who have contact with individuals requiring a protected environment, such as those who have undergone BMT. The trivalent inactivated vaccine is also indicated for healthy individuals at least 6 months or older and those with compromised immunity, and is the suitable vaccine for patients with cancer and those who have undergone BMT.

Children, elderly individuals, and younger adults all benefit from vaccination. Certain high-priority groups (e.g., patients with certain underlying diseases that place them at risk for influenza complications, children, elderly persons, pregnant women, health care workers) are targeted for public awareness campaigns each year. Table 1 provides a list of Web sites that provide updated vaccination recommendations. In addition, many state departments of health also provide information about preparedness efforts.

Unfortunately, vaccination rates in the priority groups have been less than 50\% despite the awareness efforts. Compliance with the recommendation for 2 doses of vaccine to children who are first-time vaccinees (because 1 dose does not ensure adequate protection) is also less than 50\%. ${ }^{31}$ Immunization rates for health care workers are also low. ${ }^{32}$ Clearly, improvement is needed in establishing the most effective means to attenuate the threat of influenza in the general population and cancer patients. Efforts to improve vaccination rates that are being emphasized include reminder/recall systems, standing orders for hospitalized patients, and written declination forms for health care workers. ${ }^{30}$

Immunization with inactivated vaccine early each autumn is crucial for patients with cancer. Patients, caregivers, and household contacts should be immunized. Because patients with cancer and those who have undergone BMT are immunocompromised, vaccination may not provide complete protection. ${ }^{33}$ However, vaccinating the individuals surrounding patients provides some indirect protection by reducing the likelihood of exposure. ${ }^{34,35}$

Equally important is immunizing the staff to prevent spread and reduce worker shortages. Effective vaccination programs for health care workers have been shown to be associated with a reduction in influenza outbreaks in health care facilities and a decrease in influenza-related deaths in patients. ${ }^{36-38}$

Each year, the influenza vaccine is prepared to target the likely strains deemed most likely to circulate in humans during the upcoming season. One H5N1 vaccine developed by Sanofi Pasteur, Inc. has now been FDA approved for human use; it was found to be safe and produced antibody levels expected to reduce the risk for infection in nearly half of vaccinees. Efforts to develop vaccines that produce enhanced immune responses are underway, evaluating subvirion inactivated vaccines, cold-adapted live attenuated vaccines, and adjuvants. ${ }^{39}$ These efforts are considered a high public health priority. However, vaccine shortages will probably be widespread in the event of a pandemic. Accordingly, reliance on prophylaxis with antiviral drugs will also be necessary.

\section{Isolation Procedures}

Important nonpharmacologic public health measures to prevent spread of influenza include both community and hospital measures. In the community, social distancing (avoidance of public gatherings) and school closures are important. In the pandemic of 1918 that ultimately resulted in up to 50 million deaths, 
Influenza in Oncology and BMT Units

\begin{tabular}{|c|c|c|}
\hline Web Site & Host & Information \\
\hline www.pandemicflu.gov & $\begin{array}{l}\text { Department of Health and } \\
\text { Human Services }\end{array}$ & $\begin{array}{l}\text { Guidelines for seasonal and pandemic } \\
\text { influenza preventive measures }\end{array}$ \\
\hline www.who.int & World Health Organization & $\begin{array}{l}\text { Global alerts and pandemic } \\
\text { response guidelines }\end{array}$ \\
\hline www.influenza-info.org & $\begin{array}{l}\text { Infectious Diseases Society of } \\
\text { America and Society for Healthcare } \\
\text { Epidemiology of America }\end{array}$ & $\begin{array}{l}\text { Overview of the influenza problem } \\
\text { and planning, research, and } \\
\text { treatment, with a continuing } \\
\text { education program }\end{array}$ \\
\hline$\frac{\text { www.cdc.gov/flu/professionals/ }}{\text { diagnosis/index..htm }}$ & $\begin{array}{l}\text { Centers for Disease Control and } \\
\text { Prevention }\end{array}$ & $\begin{array}{l}\text { Information about diagnostic } \\
\text { techniques }\end{array}$ \\
\hline www.idsociety.org/pandemicinfluenza.htm & $\begin{array}{l}\text { Infectious Diseases Society } \\
\text { of America }\end{array}$ & $\begin{array}{l}\text { Guidelines, news summaries, } \\
\text { and resources }\end{array}$ \\
\hline
\end{tabular}

communities that quickly closed schools, implemented steps to avoid public gatherings of people, and quarantined infected households had peak death rates that were half those of communities that did not. ${ }^{40}$ Ironically, some of this protection was lost when the safeguards were later relaxed, and infection rates climbed (but still not as high as those of communities who did not act promptly and aggressively).

Although social distancing actions will primarily necessitate public health actions, hospital and clinic facilities will need to consider similar actions. Within hospitals and clinics, avoiding overcrowded conditions, separating patients with potentially infectious illness, implementing quarantine and isolation measures for infected patients and patients suspected of being infected, emphasizing hand washing, and using masks are important procedures. Patients should avoid congregating in clinic waiting rooms and receiving chemotherapy infusions in open group settings. Separating infected patients from noninfected ones will be paramount. Visitors or family members with symptoms suggesting infection should avoid contact with cancer patients or those who have undergone BMT. Staff members must be screened daily and advised to not report to duty if they experience symptoms or signs of infection. Treatment schedule delays or delays in initiating BMT must be considered..$^{41}$

Personal protection measures must be reinforced to both staff and patients. Hand washing is key to minimizing the spread of infection because hands are a frequent means of transmission. Hand washing is important for not only staff members but also patients and their families at home. Staff should also wear gloves, gowns, and face shields when working with infected patients.

Masks are also important. Surgical masks offer some protection against droplets (believed to be the major means of transmission), but N95 respirators provide greater protection against airborne transmission. Unfortunately, no scientific data are available on which to base recommendations, but the Centers for Disease Control and Prevention (CDC) is currently recommending that, in the event of a pandemic, individuals who will be in close contact with infected patients use the N95 (or similar type) respirator. Unfortunately, a shortage of respirators is likely during a pandemic, and additional supplies will not fully address the need. In the event of shortages, surgical masks afford some protection and should be used if the more effective respirators are not available. Stockpiling additional supplies of antivirals, antibiotics, and masks is currently advisable.

\section{Antiviral Drugs}

Two classes of influenza drugs are available: adamantine drugs and neuraminidase inhibitors. The adamantine drugs, amantadine and rimantadine, are inhibitors of the M2 protein, which provides an ion channel that is important in viral replication. This drug class is active against only type $\mathrm{A}$ influenza. Unfortunately, resistance to these drugs has developed in many influenza A strains, and the $\mathrm{H} 5 \mathrm{~N} 1$ virus is not susceptible. Although the adamantine drugs have been useful for prevention and treatment of influenza in the past, these agents are less desirable today because of resistance in many of the influenza A strains now in circulation (and thus are not recommended for routine treatment or chemoprophylaxis). ${ }^{30}$ 
The neuraminidase inhibitors, oseltamivir and zanamivir, ${ }^{42}$ are active against both influenza $A$ and $B$ and exhibit activity against the $\mathrm{H} 5 \mathrm{~N} 1$ virus. Amantadine-resistant strains are generally susceptible to the neuraminidase inhibitors. The neuraminidase inhibitors have been used in cancer patients and those who have undergone BMT and were generally found to be well tolerated and effective, ${ }^{43,44}$ but systematic study has not been extensive. Although emergence of resistance has been infrequent, it has been reported in some patients who have undergone BMT. ${ }^{45}$ Mutations that confer resistance in many instances have rendered the virus less virulent. ${ }^{46}$ Furthermore, cross-resistance between the 2 neuraminidase inhibitors does not always occur. Although H5N1 isolates generally have been susceptible, sporadic resistance has also been noted. ${ }^{47}$

The neuraminidase inhibitor class of drugs is the mainstay of influenza treatment and chemoprophylaxis. In patients who are not immunocompromised, clinical trials show that these agents reduce the duration and severity of illness ${ }^{48-51}$ and reduce the frequency of serious lower respiratory tract complications..$^{52,53}$ Patients who began antiviral therapy before the onset of lower respiratory tract symptoms had a lower likelihood of developing pneumonia. ${ }^{14}$ For full effectiveness, therapy should be started within 12 to 48 hours of symptom onset. ${ }^{54,55}$ Several caveats should be mentioned for patients who are highly immunocompromised, such as those who have undergone BMT. Because viral shedding may be higher and last longer in patients who have undergone BMT, they therefore require a longer course of treatment. Continuation of therapy until resolution of symptoms and signs and cessation of shedding may be a reasonable goal. However, more studies are needed to establish the optimal dose schedule and duration in patients who are immunocompromised.

The neuraminidase inhibitors have also been shown to be effective in unimmunized healthy individuals as chemoprophylaxis for reducing seasonal influenza without the emergence of resistance. ${ }^{56-59}$ Chemoprophylaxis has not been extensively studied in patients who are immunocompromised. One trial suggested a reduction of illness in patients who have undergone BMT. ${ }^{43}$ Chemoprophylaxis should be considered in patients who are immunocompromised and are not immunized during an outbreak..$^{30}$ Because immunization may not afford protection, patients who are highly immunocompromised (even if immunized) should also be considered for chemoprophylaxis during a community outbreak. Unimmunized health care workers are also candidates for chemoprophylaxis during outbreaks. ${ }^{30}$

The CDC, Infectious Diseases Society of America, and the American Society of Blood and Marrow Transplantation have published guidelines for preventing opportunistic infections. Chemoprophylaxis is recommended for patients who have undergone BMT during community or nosocomial outbreaks less than 6 months after transplantation, or even later if they are not immunized or are highly immunocompromised from graft-versus-host disease or high doses of immunosuppressive drugs. ${ }^{35}$ When the BMT guidelines were developed, the adamantine drugs were recommended because of insufficient experience with the neuraminidase inhibitors. Today, for reasons mentioned earlier and published experience in patients who have undergone $\mathrm{BMT},{ }^{43}, 45$ neuraminidase inhibitors are preferred for chemoprophylaxis.

\section{Antibiotics}

Because of the high risk for lower respiratory tract bacterial infection, which is one of the most deadly complications of influenza, vigilance is necessary and antibiotics should be initiated when bacterial pneumonia is suspected. The need for vigilance, antiviral prophylaxis, and therapy is greater in patients who undergo allogeneic BMT than those who undergo autologous BMT because of the deeper and more prolonged immunosuppression. In the nontransplant setting, immunosuppression is typically much less, but patients receiving purine analogues (e.g., fludarabine, cladribine, pentostatin), corticosteroids, or monoclonal antibodies, such as alemtuzumab or antithymocyte globulin, also may experience profound and prolonged immunosuppression that renders them very vulnerable. The risk is also substantial in young children without antecedent immunity to influenza. Streptococcus pneumoniae and Staphylococcus aureus are the most common bacterial pathogens, and antibiotics should be chosen to treat these pathogens until a specific origin is known.

\section{Anti-Inflammatory Agents}

In H5N1 influenza, the release of cytokines and chemokines accounts for considerable morbidity. ${ }^{27}$ Corticosteroids and various anti-inflammatory agents have the potential to attenuate these deleterious host responses and may be useful adjuncts to antiviral 
Influenza in Oncology and BMT Units

therapy, but because anti-inflammatory measures may worsen the course of infection and these measures have not been systematically evaluated, controlled trials are a priority.

\section{References}

1. Adams PF, Hendershot GE, Marano MA. Current estimates from the National Health Interview Survey, 1996. Vital Health Stat 10. 1999;200:1-203.

2. Thompson WW, Shay DK, Weintraub E, et al. Mortality associated with influenza and respiratory syncytial virus in the United States. JAMA 2003;289:179-186.

3. Thompson WW, Shay DK, Weintraub E, et al. Influenza-associated hospitalizations in the United States. JAMA 2004;292:1333-1340.

4. Cox NJ, Subbarao K. Global epidemiology of influenza: past and present. Annu Rev Med 2000;51:407-421.

5. Morse SS. Pandemic influenza: studying the lessons of history. Proc Natl Acad Sci U S A 2007;104:7313-7314.

6. World Health Organization. Epidemic and pandemic alert and response (EPR). Available at: http://www.who.int/csr/disease/avian_ influenza/en/. Accessed December 17, 2007.

7. Treanor JJ. Influenza virus. In: Mandell GL, Bennett JE, Dolin R, eds. Principles and Practice of Infectious Diseases. Philadelphia: Elsevier; 2005:2060-2085.

8. Peck AJ, Englund JA, Kuypers J, et al. Respiratory virus infection among hematopoietic cell transplantation recipients: evidence for asymptomatic parainfluenza virus infection. Blood 2007;110: 1681-1688.

9. Hicks KL, Chemaly RF, Kontoyiannis DP. Common community respiratory viruses in patients with cancer: more than just "common colds". Cancer 2003;97:2576-2587.

10. Ison MG, Hayden FG. Viral infections in immunocompromised patients: what's new with respiratory viruses? Curr Opin Infect Dis 2002;15:355-367.

11. Whimbey E, Englund JA, Couch RB. Community respiratory virus infections in immunocompromised patients with cancer. Am J Med 1997;102:10-18.

12. Whimbey E, Elting LS, Couch RB, et al. Influenza A virus infections among hospitalized adult bone marrow transplant recipients. Bone Marrow Transplant 1994;13:437-440.

13. Schepetiuk S, Papanaoum K, Qiao M. Spread of influenza A virus infection in hospitalised patients with cancer. Aust N Z J Med 1998;28:475-476.

14. Chemaly RF, Ghosh S, Bodey GP, et al. Respiratory viral infections in adults with hematologic malignancies and human stem cell transplantation recipients: a retrospective study at a major cancer center. Medicine (Baltimore) 2006;85:278-287.

15. Kempe A, Hall CB, MacDonald NE, et al. Influenza in children with cancer. J Pediatr 1989;115:33-39.

16. Whimbey E, Champlin RE, Couch RB, et al. Community respiratory virus infections among hospitalized adult bone marrow transplant recipients. Clin Infect Dis 1996;22:778-782.

17. Arola $M$, Ruuskanen $O$, Ziegler $T$, et al. Respiratory virus infections during anticancer treatment in children. Pediatr Infect Dis J 1995; 14:690-694.

18. Yousuf HM, Englund J, Couch R, et al. Influenza among hospitalized adults with leukemia. Clin Infect Dis 1997;24:1095-1099.
19. Long CE, McBride JT, Hall CB. Sequelae of respiratory syncytial virus infections. A role for intervention studies. Am J Respir Crit Care Med 1995;151:1678-1680.

20. Erard V, Chien JW, Kim HW, et al. Airflow decline after myeloablative allogeneic hematopoietic cell transplantation: the role of community respiratory viruses. J Infect Dis 2006;193:1619-1625.

21. Elting LS, Whimbey E, Lo W, et al. Epidemiology of influenza $A$ virus infection in patients with acute or chronic leukemia. Support Care Cancer 1995;3:198-202.

22. Couch RB, Englund JA, Whimbey E. Respiratory viral infections in immunocompetent and immunocompromised persons. Am J Med 1997;102:2-9.

23. Nichols WG, Guthrie KA, Corey L, et al. Influenza infections after hematopoietic stem cell transplantation: risk factors, mortality, and the effect of antiviral therapy. Clin Infect Dis 2004;39:1300-1306.

24. Boivin G, Hardy I, Tellier G, et al. Predicting influenza infections during epidemics with use of a clinical case definition. Clin Infect Dis 2000;31:1166-1169.

25. Beigel JH, Farrar J, Han AM, et al. Avian influenza A (H5N1) infection in humans. N Engl J Med 2005;353:1374-1385.

26. Yuen KY, Chan PK, Peiris M, et al. Clinical features and rapid viral diagnosis of human disease associated with avian influenza A H5N1 virus. Lancet 1998;351:467-471.

27. de Jong MD, Simmons CP, Thanh TT et al. Fatal outcome of human influenza A (H5N1) is associated with high viral load and hypercytokinemia. Nat Med 2006;12:1203-1207.

28. Tsang KW, Shim YS, Wong TK, et al. Possible case scenarios and logistic issues in H5N1 pandemic. Respirology 2006;11:520-522.

29. Wingard JR, Leahigh AK, Confer D, et al. Preparing for the unthinkable: emergency preparedness for the hematopoietic cell transplant program. Biol Blood Marrow Transplant 2006;12:1229-1238.

30. Fiore AE, Shay DK, Haber P, et al. Prevention and control of influenza. Recommendations of the Advisory Committee on Immunization Practices (ACIP), 2007. MMWR Recomm Rep 2007; $56: 1-54$.

31. Jackson LA, Neuzil KM, Baggs J, et al. Compliance with the recommendations for 2 doses of trivalent inactivated influenza vaccine in children less than 9 years of age receiving influenza vaccine for the first time: a Vaccine Safety Datalink study. Pediatrics 2006;118: 2032-2037.

32. Esposito S, Tremolati E, Bellasio M, et al. Attitudes and knowledge regarding influenza vaccination among hospital health workers caring for women and children. Vaccine 2007;25:5283-5289.

33. Gross PA, Gould AL, Brown AE. Effect of cancer chemotherapy on the immune response to influenza virus vaccine: review of published studies. Rev Infect Dis 1985;7:613-618.

34. Machado CM, Cardoso MR, da Rocha IF, et al. The benefit of influenza vaccination after bone marrow transplantation. Bone Marrow Transplant 2005;36:897-900.

35. Centers for Disease Control and Prevention; Infectious Disease Society of America; American Society of Blood and Marrow Transplantation. Guidelines for preventing opportunistic infections among hematopoietic stem cell transplant recipients. MMWR Recomm Rep 2000;49: 1-125, CE1-7. Erratum in MMWR Recomm Rep 2004;53:396.

36. Wilde JA, McMillan JA, Serwint J, et al. Effectiveness of influenza vaccine in health care professionals: a randomized trial. JAMA 1999;281:908-913.

37. Carman WF, Elder AG, Wallace LA, et al. Effects of influenza vaccination of health-care workers on mortality of elderly people in long-term care: a randomised controlled trial. Lancet 2000;355:93-97. 
38. Potter J, Stott DJ, Roberts MA, et al. Influenza vaccination of health care workers in long-term-care hospitals reduces the mortality of elderly patients. J Infect Dis 1997;175:1-6.

39. Nichol KL, Treanor JJ. Vaccines for seasonal and pandemic influenza. J Infect Dis 2006;194(Suppl 2):S111-118.

40. Hatchett RJ, Mecher CE, Lipsitch M. Public health interventions and epidemic intensity during the 1918 influenza pandemic. Proc Natl Acad Sci U S A 2007;104:7582-7587.

41. Kumar D, Humar A. Pandemic influenza and its implications for transplantation. Am J Transplant 2006;6:1512-1517.

42. Baum SG. Oseltamivir and the influenza alphabet. Clin Infect Dis 2006;43:445-446.

43. Vu D, Peck AJ, Nichols WG, et al. Safety and tolerability of oseltamivir prophylaxis in hematopoietic stem cell transplant recipients: a retrospective case-control study. Clin Infect Dis 2007;45: 187-193.

44. Johny AA, Clark A, Price N, et al. The use of zanamivir to treat influenza A and B infection after allogeneic stem cell transplantation. Bone Marrow Transplant 2002;29:113-115.

45. Machado CM, Boas LS, Mendes AV, et al. Use of Oseltamivir to control influenza complications after bone marrow transplantation. Bone Marrow Transplant 2004;34:111-114.

46. Hayden FG. Antivirals for influenza: historical perspectives and lessons learned. Antiviral Res 2006;71:372-378.

47. Moscona A. Oseltamivir resistance-disabling our influenza defenses. N Engl J Med 2005;353:2633-2636.

48. Nicholson KG, Aoki FY, Osterhaus AD, et al. Efficacy and safety of oseltamivir in treatment of acute influenza: a randomised controlled trial. Neuraminidase Inhibitor Flu Treatment Investigator Group. Lancet 2000;355:1845-1850.

49. Hayden FG, Osterhaus AD, Treanor JJ, et al. Efficacy and safety of the neuraminidase inhibitor zanamivir in the treatment of influenzavirus infections. GG167 Influenza Study Group. N Engl J Med 1997; 337:874-880,

50. Moscona A. Neuraminidase inhibitors for influenza. N Engl J Med 2005;353:1363-1373.

51. Monto AS, Fleming DM, Henry D, et al. Efficacy and safety of the neuraminidase inhibitor zanamivir in the treatment of influenza $A$ and B virus infections. J Infect Dis 1999;180:254-261.

52. Kaiser L, Wat C, Mills T, et al. Impact of oseltamivir treatment on influenza-related lower respiratory tract complications and hospitalizations. Arch Intern Med 2003;163:1667-1672.

53. Kaiser L, Keene ON, Hammond JM, et al. Impact of zanamivir on antibiotic use for respiratory events following acute influenza in adolescents and adults. Arch Intern Med 2000;160:3234-3240.

54. Treanor JJ, Hayden FG, Vrooman PS, et al. Efficacy and safety of the oral neuraminidase inhibitor oseltamivir in treating acute influenza: a randomized controlled trial. US Oral Neuraminidase Study Group. JAMA 2000;283:1016-1024.

55. Aoki FY, Macleod MD, Paggiaro P, et al. Early administration of oral oseltamivir increases the benefits of influenza treatment. J Antimicrob Chemother 2003;51:123-129.

56. Hayden FG, Atmar RL, Schilling M, et al. Use of the selective oral neuraminidase inhibitor oseltamivir to prevent influenza. N Engl J Med 1999;341:1336-1343.

57. Monto AS, Robinson DP, Herlocher ML, et al. Zanamivir in the prevention of influenza among healthy adults: a randomized controlled trial. JAMA 1999;282:31-35.

58. Welliver R, Monto AS, Carewicz O, et al. Effectiveness of oseltamivir in preventing influenza in household contacts: a randomized controlled trial. JAMA 2001;285:748-754.

59. Hayden FG, Belshe R, Villanueva C, et al. Management of influenza in households: a prospective, randomized comparison of oseltamivir treatment with or without postexposure prophylaxis. J Infect Dis 2004;189:440-449. 